\title{
BREMSSTRAHILUNG FrOM HIGH Z IMPURITIES IN HOT PLASMAS
}

\author{
Hugh Dewitt, Forrest Rogers, and Carlos Iglesias \\ Lawrence Livermore National Laboratory \\ $\checkmark$ Division, Physics Department \\ Livermore, Californta 94550
}

LCID- -21403

DI98 011755

\section{ABSTRACT}

This is a study of the effect of core electron and plasma scrioning on Bremsstrahlung from high $Z$ impurities in hot plasmas.

\section{DISCLAIMER}

This report was prepared as an account of work sponsored by an agency of the United States Government. Neither the United Slates Government nor any ggency thereof, not any of tbeir employees, makes any warranty, express or implied, of assumes any legal liability or responsibility for the accuracy, completeness, or usefulness of any information, apparatus, product, or process dieclosed, or represents that its use would not infringe privately owned rights. Reference herein to any specific commercial product, process, or service by trade name, trademark. manufacturer, or otherwise does not necessarily constitute or imply its endorsement, recommendation, or favoring by the United States Government of any agency thereof. The views and opinions of autbors expressed herein do not necessarily state or reflect those of the United States Government or any agency thereof. 
The purpose of this report is to discuss some quantitative results on the Bremsstrahiung spectrum from high $Z$ impurtties in ho' plasmas. The main goals of this study were to find out quantitatively (1) the effect of free particle screening on different regions of the Bremsstrahlung spectrum, and (ii) the effect of core electrons bound to the high $Z$ nucleus. Also, we wanted to assess the utility of the coupled HNC equations for generation an electron-ion scattering potential and the Gaunt factor code that forms part of the new opacity code recently developed by Rogers and Iglesias. The discussion here is limited to Case III (of the seven cases Tommy Thompson gave us on Feb. 10), namely: density $=1.0 \mathrm{gm} / \mathrm{cc}$ with .9995 hydrogen and $.0005 \mathrm{xe}$ at $k T=.06 \mathrm{keV}$. Our HNC code had to deal with point electrons, point protons, and $\mathrm{xe}$ fons, i.e. at least three components. In principle, our HNC codes can handle up to four components but for computational reasons at this time, we preferred to deal anly with one heavy fon component which was chosen as $x e^{+43}$, thus 11 bound electrons around a nucleus of $z=54$. Although the Xe abundance in the hydrogenic plasma is quite sma11, the effective charge, $z^{*}=43$, is large enough that it can significantly modify the total Bremsstrahlung. Furthermore, the Gaunt factor for Xe is expected to be numerically very different from what is calculated by XSN.

It is first necessary to specify in some detail how the electron-ion potential is obtained from the coupled HNC equations. The steps are as follows: 
- 3 -

1) An effective electron-ion potential is chosen using constants obtained in Ref. 1.

$$
u_{e i}(r)=-\frac{1}{r}\left[(z-t)+\sum_{n=1}^{n} v_{n} e^{-\alpha_{n} r}\right]
$$

The first term is the coulomb potential for $z^{*}=2-r=54-11=$ 43; this is used in XSN. For the sodfum-like configuration the number of electrons in the core shells are 2, 8, and 1. The screening constants are chosen so that experimental energy levels can be reproduced.

2) Bound and scattering state wave functions are obtained by solving the Schrodinger equation for $u_{e i}(r)$.

3) As described in Ref. 2 the Slater sum is calculated and separated into pieces according to the Planck-Lark in decomposition. A plasma pseudo-potential is calculated from the quast-free state part of the slater sum:

$$
\begin{aligned}
& \beta u_{\text {er, psento }}{ }^{(r)}=-\ln \mathrm{W}_{\mathrm{ei}}^{\mathrm{I}}
\end{aligned}
$$

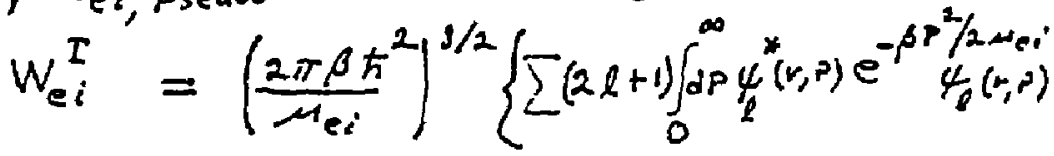

$$
\begin{aligned}
& \left.-\sum_{n, l}(2 l+1) \Psi_{n l}^{*}(r)\left(1-\beta E_{n \ell}\right) \Psi_{n \ell}(r)\right\}
\end{aligned}
$$


The generation of this pseudo-potential requires a considerable amount of computational effort, and previous numerical work (in Ref. 2) had been limited to argon. At long distance this pseudo-potential reduces to $-2^{\star} / r$, but at short distances it bullds in extensive quantum effects and at $r=0$ it goes to a finite value.

4) The HNC equations that couple point electrons, point protons, and $\mathrm{Xe}^{+43}$ tons are solved numerically using pseudo $\mathrm{U}_{\mathrm{e}}$. These are classtcal equations so that it is essential that the pseudo potentials be finite at $r=0$. This is guaranteed by wave mechanics. Otherwise the classical $-1 / r$ divergence would make solutions impossible. From the HNC solutions one obtains the various pair correlations functions, $h_{e e}(r), h_{e j}(r), h_{i j}(r)$, and their Fourier transforms (structure factors). Since the core states are already occupied, the pseudo potential created here does not have these states in the slater sum. The effect of the core electrons, however, are still present in the wave functions obtained from the effective potential, Eq. 1 .

5) The Poisson equation is formed using the pair correlation functions from the HHC solutions to give the charge densities zround a $\mathrm{Ke}^{+43}$ ion: 
$\nabla^{2} V_{e i}^{s}=-4 \pi e^{2}\left\{Z_{i} \delta(r)+\sum_{j} z_{j} \rho_{j} h_{i j}\right\}$

This equation is Fourier analyzed and solved in $k$ space (for detalls see Eqs. 45 to 48 in Ref. 2). In the case of weak coupling, it will turn out that after a short distance from the nucleus $v_{e j}^{s}(r)$ has the Yukawa form with a screening length close to, but not exactly, the same as the Debye length.

$$
V_{e i}^{s}(r)=-\frac{z^{2}}{r} e^{-r / D}
$$

where 0 is the effective screening length. In more strongly coupled plasmas, 1.e., higher density of the high $z$ impurities, the $v_{e i}^{\delta}$ will become much different from the Yukawa form, and can even become oscillatory. 
6) The core electron terms are added to $v_{e j}^{S}(r)$ to get the needed ton electron potential:

$$
V_{e i}(r)=-\frac{1}{r}\left\{(z-z) e^{-r / D}+\sum_{n=1}^{n} \nu_{n} e^{-\alpha_{n} r}\right\}
$$

Since $0>1 / \alpha_{n}$ we have Ignorer. the small plasma screening effects on the $\alpha_{n}$.

Generation of $V_{e f}(r)$ by this process may seen unduly complicated. However, it is physically motivated at every step, and we believe that it gives the best avaflable physical potential felt by an electron scattering off a high 2 ion in the presence of both core electrons and free charges. This potential is used in the free-free Gaunt factor code developed by $\mathrm{J}$. Green. Green's program can take any form of screened potential, solve the Schroedinger equation to obtain partial waves, compute the free-free dipole matrix elements in the acceleration form (which uses the gradient of $\left.v_{e f}(r)\right)$, and finally sum the squared matrix elements over enough partial waves to give the quantum mechanical result to any desired accuracy. The result is the Gaunt factor as a function of the momentum of the scattering electron. Finally, the electron momenta are averaged over a Maxwellian distribution to give the Gaunt factor for the appropriate temperature. The 
momentum averaging process turns out to be exactly the same as that used by $B$. Rozsnyaf in his HOPE code. Dur $V_{e f}(r)$ is somewhat different from the electron-ion potential generated in HOPE. Rozsnyaf's potential is more screened than aurs because he fits a superposition of two Yukawa potentials to the ion-sphere potential which is neutral at the fon-sphere radius. We believe that hs treatement of core electrons is inadequate when the core involves $M$ shell electrons, or higher shells.

Figure 1 shows a plot of $-x v_{e j}(x)$ for the $x e^{+43}$ potential (upper line) as compared with the Debye Huckel potential (lower line). The actual screening length from the HNC calculation is $0=4.585$ bohr whereas the Debye length is 4.09 or about $11 \times$ lower. The three Yukawe. terms for the core electrons are included in these plots. The inverse screening lengths for the $K$. $L$, and $M$ shells are respectively $41.612,16.531$, and 6.4462 in units of 1/bohr. Note that $1 / 0=.2181 /$ bohr. The core electrons are pulled in very close to the $X e$ nucleus as compared with the screening due to free charges. Figure 2 shows a close up plot of the short distance part of the potential. the core electron effect starts to show up at about .03 bohr.

Figure 3 shows a comparison of the Guant factor for $Z=1$ from $X S N$ and from our quantum mechanical calculation. This is basically a comparison of a complete non-relativistic quantum dipole calculation of the Gaunt factor for the pure coulomb potential (XSN) vs our result for a simple Yukawa screened potentlal. As expected, screening effects are negligible for photons above 1 Kev, but give about a $6 \%$ reduction at $.7 \mathrm{KeV}$. In order to demonstrate the relative importance of different screening lengths we ran the Gaunt factor 
calculation for three different screening lengths, 20, 0 (the physical value, 4.59 bohr), and $0 / 2$. Again as expected all three curves on Figure 4 converge above $1 \mathrm{KeV}$, and show very marked differences at $.1 \mathrm{KeV}$ due to the different srreening lengths.

For the Gaunt factor calculations using the electron-ion potential, eq. 5, we can use the full potential with the core electrons or turn off the core and simply use the screened coulomb part with $Z^{*}=43$. In this way we can explicitly show the effects of the core electrons. Figure 5 shows the comparison of the two calculations with XSN. This is the main result presented in this memo. The numerical data is given in Table $I$. At low photon energy, 0.1 Kev, the curves with the core and with no core converge and are about 30\% lower than the XSN value because of screening. The low energy photons are emitted by electrons that scatter at some distance from the core and thus see a screened potential with $z^{\star}=43$. At the high energy photon end, $10 \mathrm{KeV}$, the screened Gaunt factor should approach the XSN result, as was the case for $Z=1$ shown in Figure 3 . In fact the 'no core' curve does lie below the $X S H$ value, but remains about $3 \%$ low. This is something we would not have discovered without trying the $J$. Green code out for these high values of $Z^{*}$. Of more importance is the Gaunt factor calculation with the core electrons. 
The important and obvious point is that the core around the $x e$ nucleus seriously affects the Gaunt factor value and leads to about a $30 \%$ increase over the XSN value. Physically this is due to very fast eiectrons which can penetrate the core and see not $Z^{*}=43$, but a higher value of $Z$ approaching $Z=54$. The calculation here is non-relativistic but that should not matter since the relativistic correction in XSN for $10 \mathrm{kev}$ photons amounts to only a $1 \%$ increase in the Gaunt factor.

Figure 6 shows the Gaunt factors for three different screening lengths. Again screening makes! a very significant difference at the low frequency end of the spectrum, and a 11 three curves converge at the hign frequency end where screening is unimportant but core penetration is important. Figure 7 shows a similar study of the Gaunt factors for three screening lengths but with no core. The three curves converge at high frequency to a value slightly lower than the XSN value.

B. Rozsnyai also ran the HOPE potential through his Gaunt factor calculation both with and without his core term (a single Yukawa), and found results similar to our figure 5. At high frequency his Guant factor with no core also approached XSH from below as expected, but with the core included 
his result was significantly above XSN. Since his potential is different from ours, we will not include his results on our figures. The purpose here is only to report results of a systematic study on the highest density xe case (Case III). reguested by $\mathrm{T}$. Thomson.

CONCLUSIONS:

A. The high frequency Bremsstrahlung spectrum may be increased by as much as $30 \%$ above XSN due to fast electrons penetrating the core of bound electrons.

B. Screening reduces Bremsstrahlung by a few percent in the $1 \mathrm{keV}$ region, 20 to $30 \%$ in the $.1 \mathrm{keV}$ region, and hardly at all at $10 \mathrm{keV}$.

\section{REFERENCES}

1. F. J. Rogers, Phys. Rev. A23, 1008 (1981)

2. F. J. Rogers, Phys. Rev. A29, 868 (1984)

3. Joseph Green, RDA-TR-4900-007 (1973) 
Table I

Gaunt Factor Data, QM with Screening, and XSN

$\begin{array}{ccccc}\begin{array}{c}h Z \\ \text { in keV }\end{array} & \begin{array}{c}\text { with } \\ \text { core }\end{array} & \begin{array}{l}\text { no } \\ \text { core }\end{array} & \begin{array}{l}\text { hz } \\ \text { in keV }\end{array} & \text { XSN } \\ 0.060 & 1.0331 & 1.0221 & 0.100 & 1.285 \\ 0.150 & 1.0879 & 1.0731 & 0.158 & 1.230 \\ 0.360 & 1.1037 & 1.0744 & 0.251 & 1.187 \\ 0.600 & 1.1168 & 1.0707 & 0.398 & 1.156 \\ 1.200 & 1.1525 & 1.0683 & 0.631 & 1.133 \\ 1.800 & 1.1856 & 1.0691 & 1.000 & 1.118 \\ 2.400 & 1.2145 & 1.0704 & 1.585 & 1.109 \\ 3.000 & 1.2402 & 1.0721 & 2.512 & 1.105 \\ 3.600 & 1.2630 & 1.0737 & 3.987 & 1.105 \\ 5.400 & 1.3186 & 1.0779 & 6.310 & 1.109 \\ 7.200 & 1.3611 & 1.0812 & 70.000 & 1.116 \\ 9.600 & 1.4049 & 1.0848 & & \\ 12.000 & 1.4389 & 1.0874 & & \end{array}$




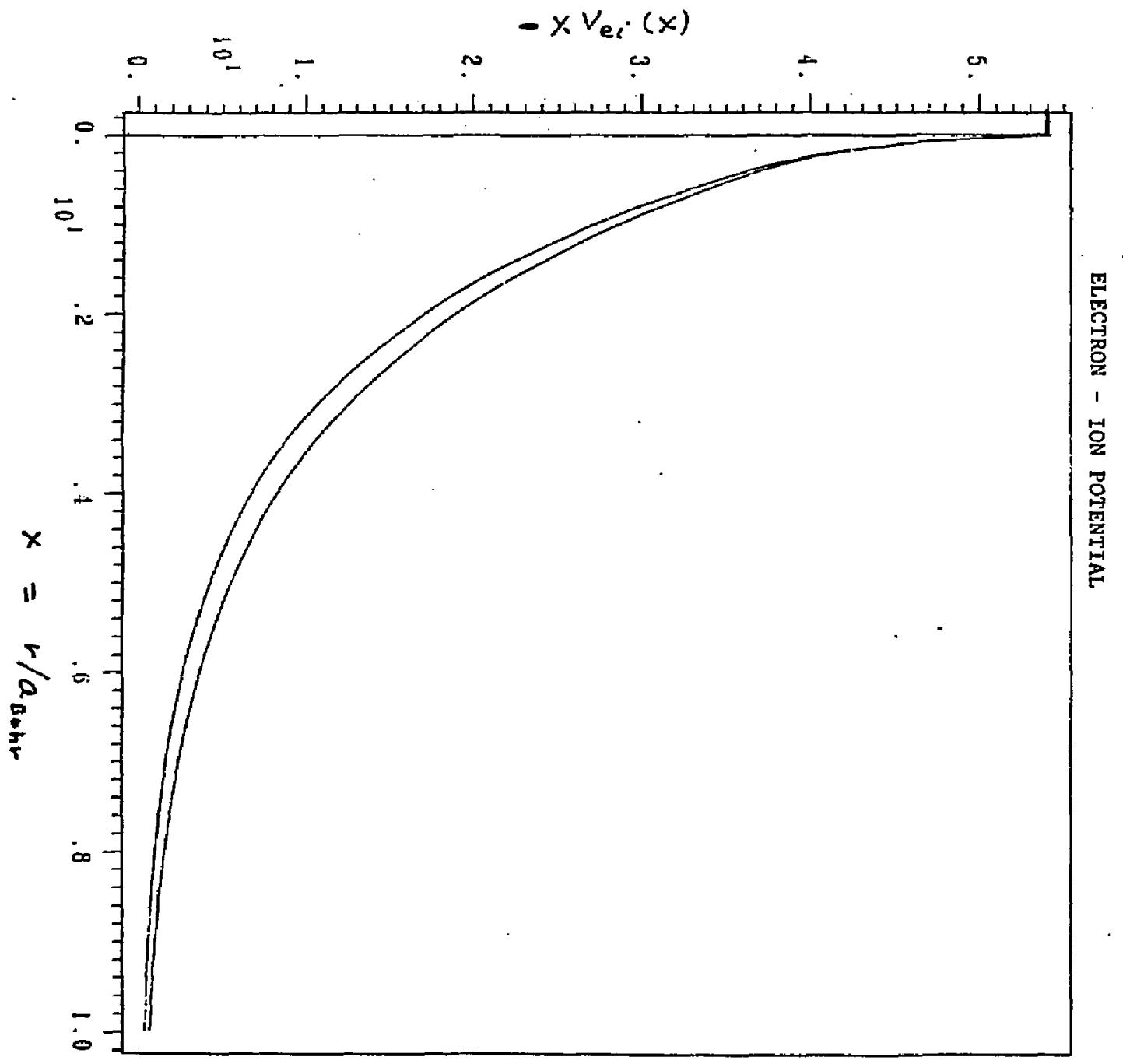

Fig. 1. Electron-Xe $e^{+43}$ potential. The upper line gives the actual result obtalned from the coupled HNC equations with the- three Yukawa terms. added to give the core electron effects. The lower curve is the result with the Debye screening length plus the core electron terms. The Debye length is about 117 less than the screening length, $D$, obtained from the HNC calculations. 


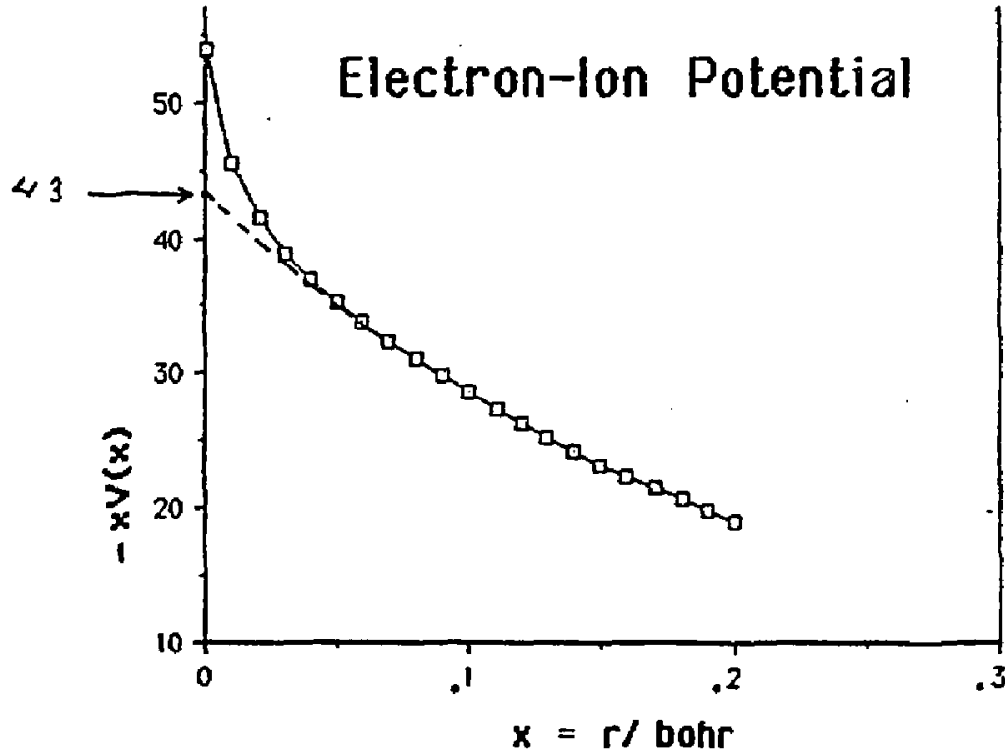

Fig. 2 A Short Distance Close-up of the Electron-Xe ${ }^{+43}$ Potential to Show the Core Electron Effects. Without the core electron terms the screened potential corresponds to $\mathrm{z}^{\star}=43$. 


\section{Gaunt Factor for $\mathrm{Z}=1$ from XSN and Screened Potential}

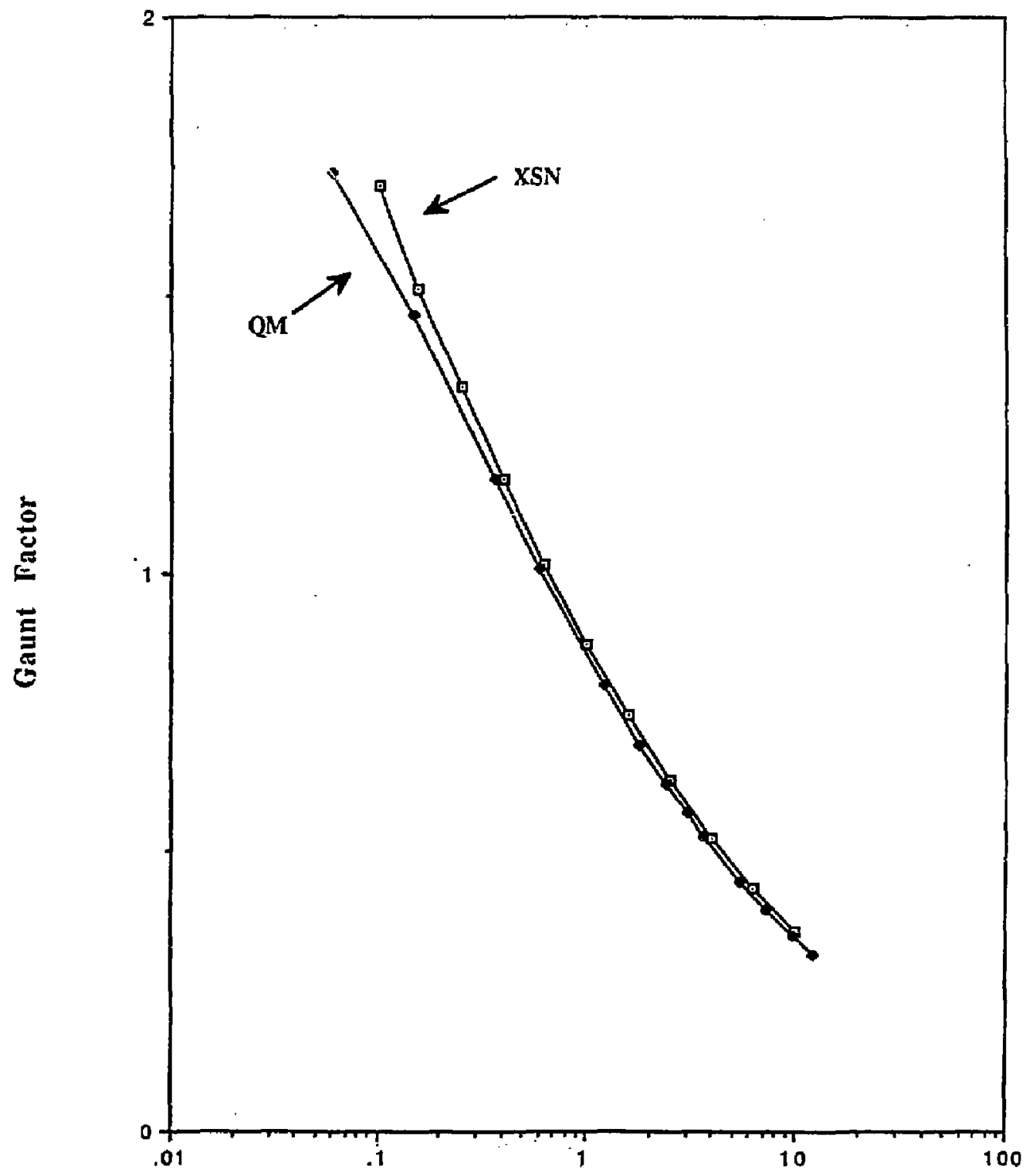

\section{Photon Energy in keV}

Fig. 3 Gaunt Factors for $z=1$. The upper curve is from XSN, 1.e. pure Coulomb The lower curve is our result obtalned from the HNC potential with screening length $D$. Our $Q M$ calculation should be of the same accuracy as the XSN result, hence the lower curve must be very close to XSN above $1 \mathrm{Kev}$ where screening effects are negligible. 


\section{Gaunt Factor for $\mathrm{Z}=1$ with Different Screening Lengths}

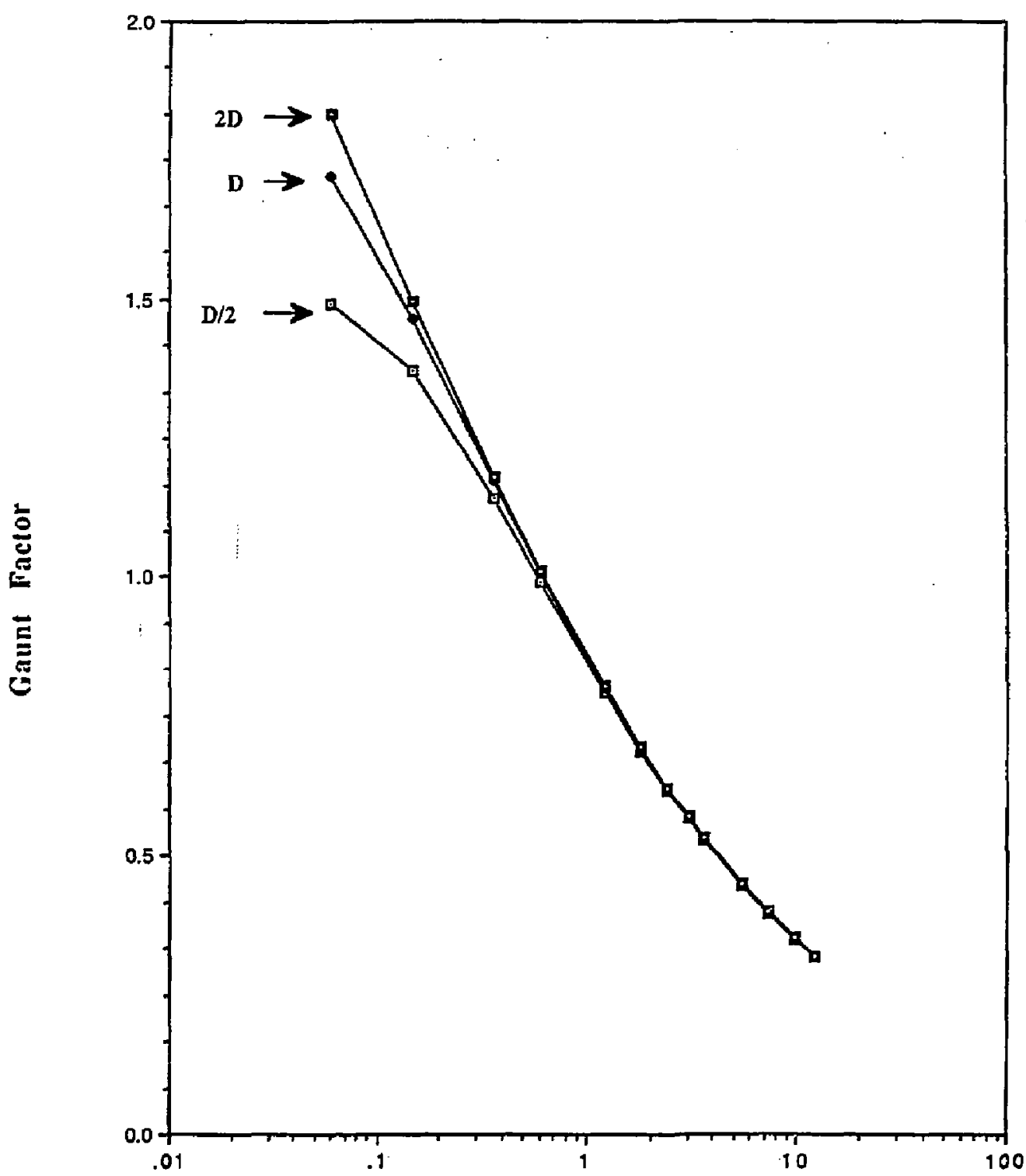

Photon Energy in keV

Fig. 4 Gaunt Factors for Three Screenting Lengths. As experted the three curves converge above $1 \mathrm{KeV}$, but screening effects are sizeable at . $1 \mathrm{Kev}$. 


\section{Gaunt Factor with Core and No Core}

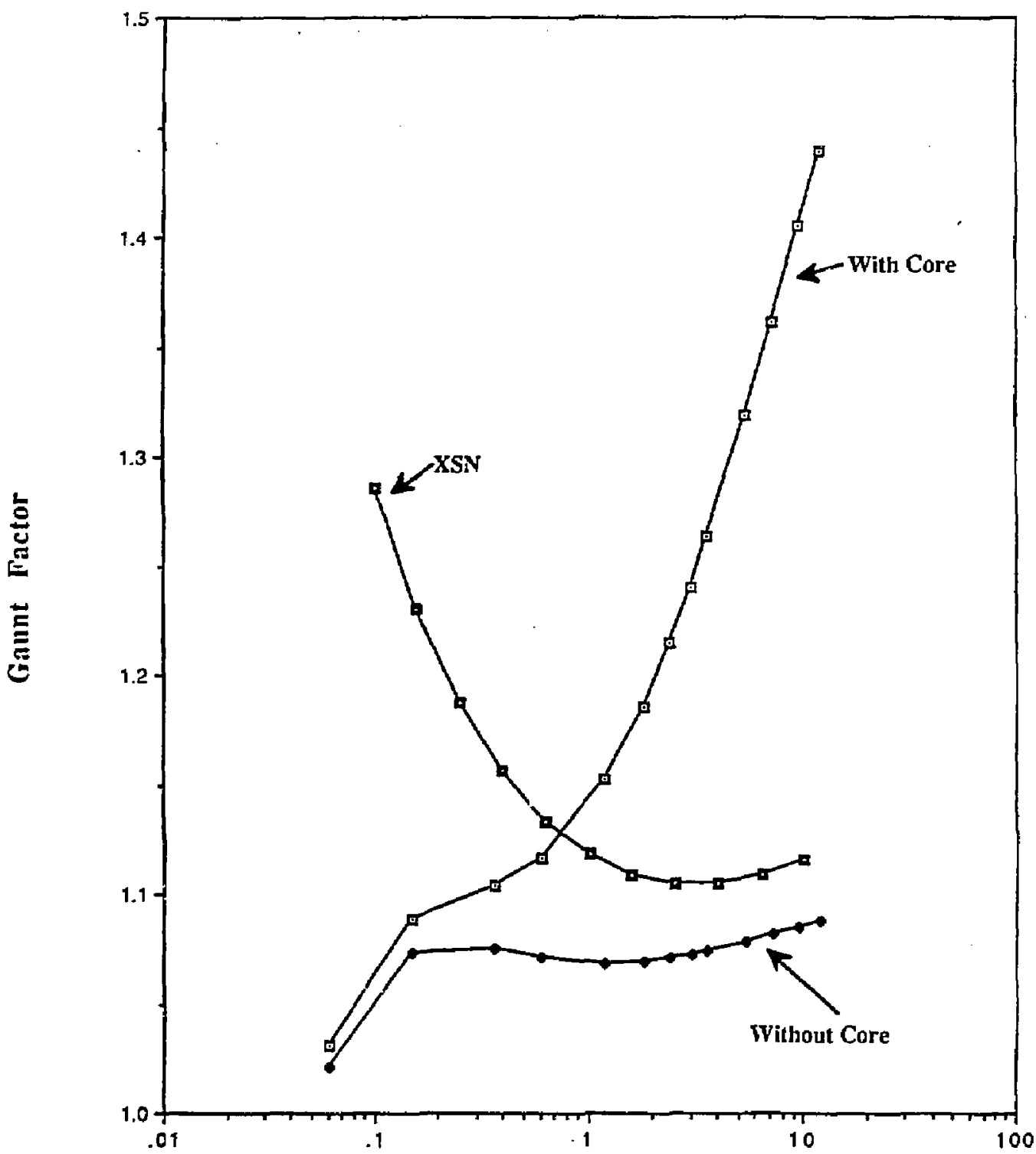

Photon Energy in keV

F1g. 5 The Ma1n Result of This Report. The upper curve gives the Gaunt factor from the QM calculation using the HNC potential plus three Yukawa terms for the core electrong. The lower curve is the same calculation with no core electrons, $\mathrm{z}=43$. Both curves converge at low frequency and show the screening effect. 


\section{Gaunt Factor with Core \\ with Different Screening Lengths}

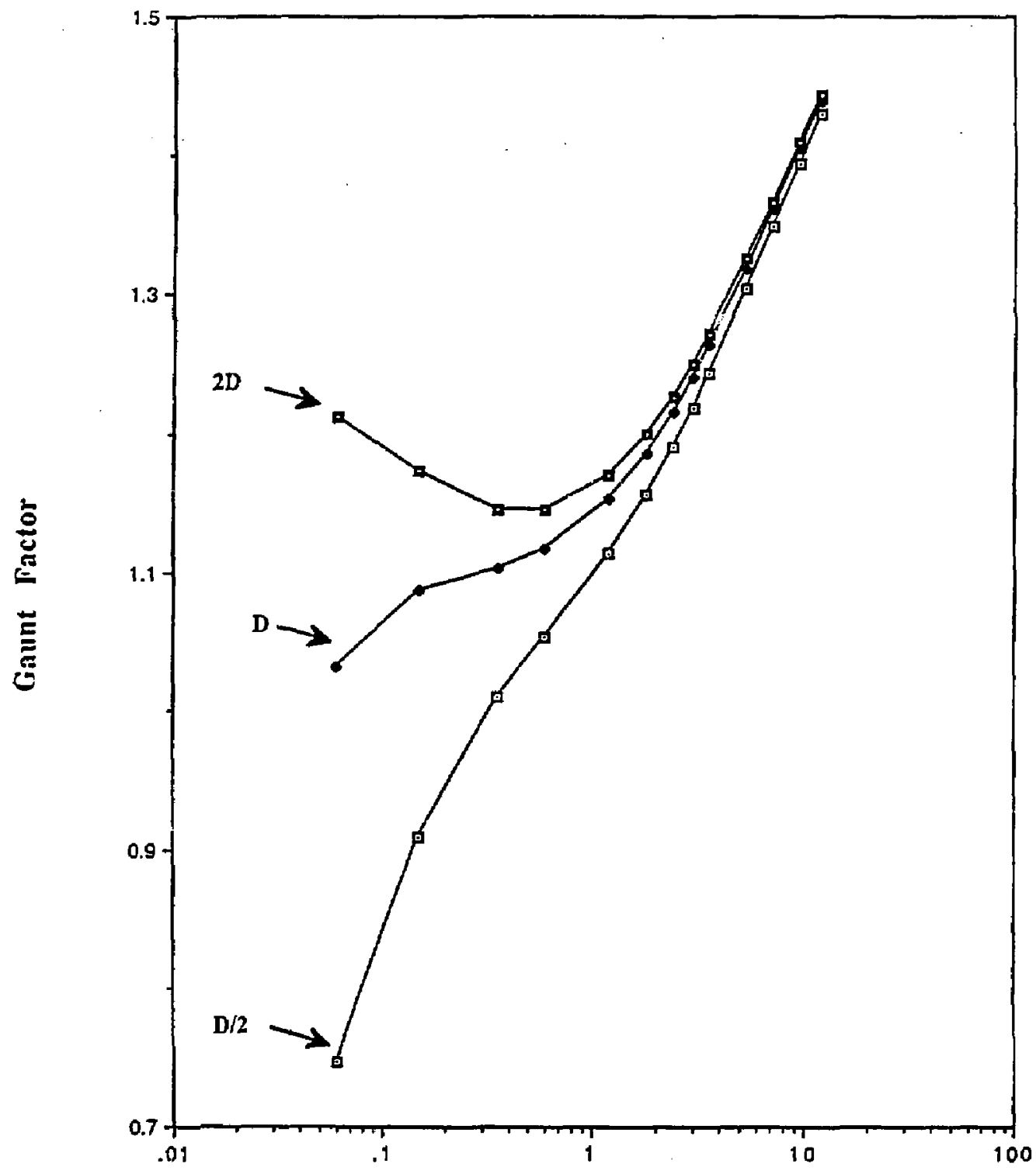

Photon Energy in keV

Fig. 6 The QM Gaunt Factor Results for Three Different Screening Lengths for Xe $\mathrm{Xe}^{+43}$. All three curves converge at high frequency indicating the same result for the electron core effects. 
Gaunt Factor for $\mathrm{Z}=43$ with Different
Screening Lengths Compared with XSN

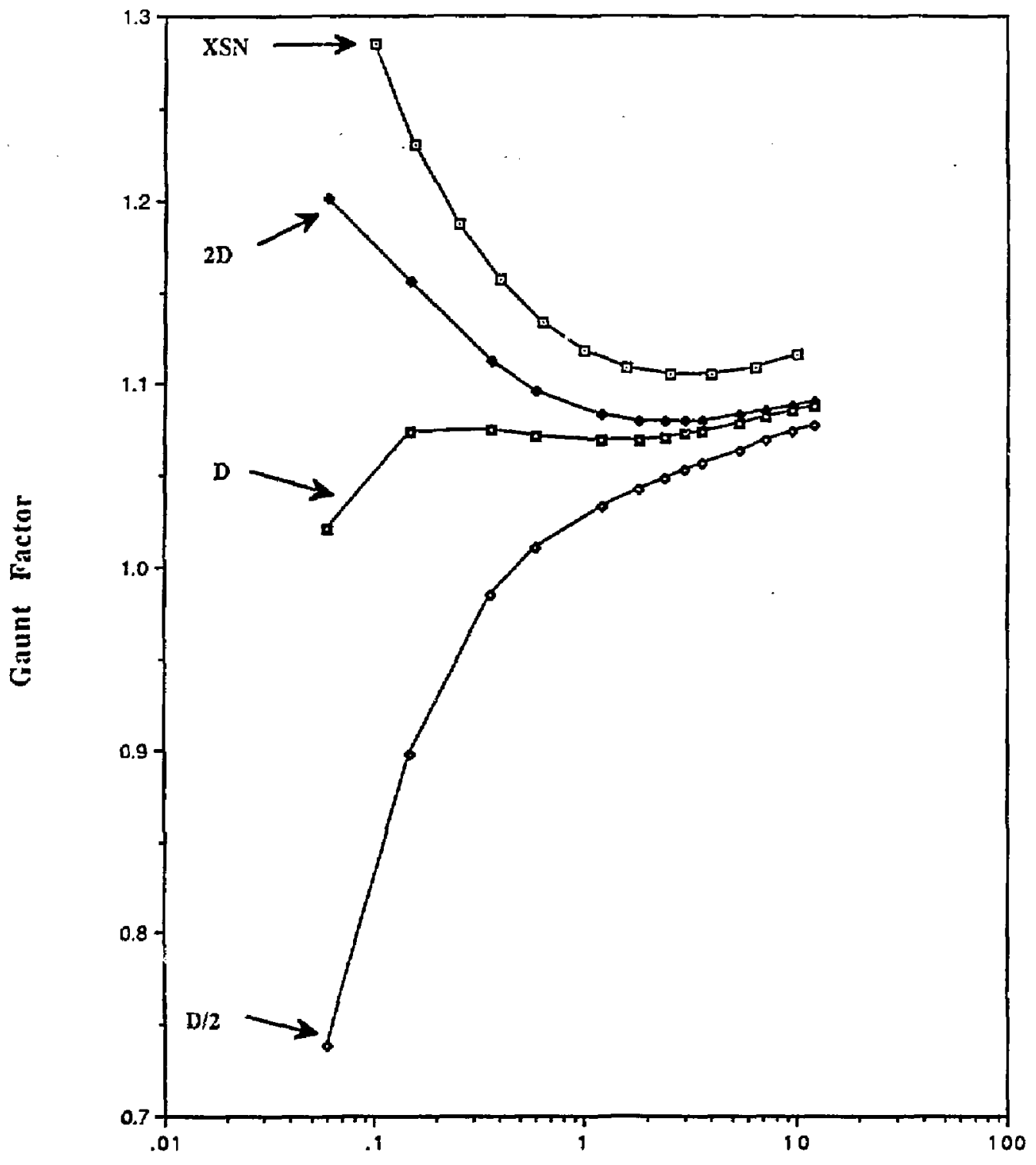

Photon Energy in keV

Fig. 7. Gaunt Factors with No Core Electrons, $z^{*}=43$, Compared with XSN. All three curves for the different screening lengths converge to the same point about $3 \%$ below the high frequency XSN result. 
Distribution:

David Bailey

Charles Cerjan

David Dearborn

Hugh Dewitt

Harold Graboske

Andy Hazi

Carlos Iglesias

Dick More

10

Burke Ritchie

Forrest Rogers

Balazs Rozsnyai

10

Tom Thomson

Tom Weaver

George Zimmerman 